\title{
Factors determining maximum torque and achievement of the recommended torque for manual implant drivers: A pilot study
}

\author{
Tomoki Nishiuchi, Yuji Sato, Noboru Kitagawa, \\ Tokiko Osawa, Akio Isobe* and Hiroki Shiba
}

Received: 21 December 2020 / Accepted: 28 February 2021

\begin{abstract}
When fixing an oral implant superstructure with a screw, operators must be aware of the torque being applied by their fingers to prevent the transmission of excessive or insufficient torque to the implant. In this study, we identified the factors that determine individual maximum attainable torque and those that determine the achievement of the prescribed torque. We evaluated 16 dentists on their use of two types of manual implant drivers (UniGrip by Nobel Biocare and Carrier Hex by Zimmer Biomet) and measured the maximum torque (MT) generated by their fingers. The target torque was set at $15 \mathrm{~N}$. Measurements were taken while the participants were turning the implant screw with or without gloves in both clockwise and counterclockwise directions. The grip and finger strength of each participant were measured, and the data showed that torque values were higher among the male participants during clockwise rotation and when they were wearing gloves $(p<0.05)$. Positive correlations were found between the MT and grip strength and between the MT and finger strength. These results suggest that dentists should monitor their ability to consistently achieve the recommended torque for implant drivers.
\end{abstract}

Key words : manual driver, maximum torque, implant

\section{Introduction}

Oral implants are widely used for the treatment of patients with an edentulous jaw or partial loss of teeth ${ }^{1-3}$. The demand for implant treatment is expected to increase and will become a key part of dental practice as society ages. However, outstanding issues still need to be addressed to increase their success rate. One of these issues is the mechanical force applied to the implant superstructure. Distortions of implant superstructures may loosen or fracture the screws in resin and porcelain veneers, whereas those in the surrounding bone tissue can lead to damage of the bone surrounding the implant ${ }^{4-7}$. One of the most commonly reported complications in implant treatment is screw loosening or fracture ${ }^{8,9}$, which is believed to be caused by

\footnotetext{
* Corresponding author

$凶$ Akio Isobe

isobeaki@dent.showa-u.ac.jp
}

Department of Geriatric Dentistry, School of Dentistry, Showa University, 2-1-1 Kitasenzoku, Ota-ku, Tokyo 1458515, Japan. implant superstructure incompatibility, repeated bending moments, insufficient initial tightening of the screw, insufficient screw strength, insufficient tightening force applied to the screw, or excessive tightening force applied to the screw ${ }^{10}$. In this study, we focused on the application of insufficient or excessive tightening force as a risk factor for screw loosening or fracture. The recommended strategy for fixing an implant superstructure is for the screw to first be manually tightened before being tightened with a torque wrench following the manufacturer's prescribed torque. Several scenarios make the use of a torque wrench difficult, namely, deterioration of the torque wrench ${ }^{11}$, limited mouth opening in patients, and tightening of cover screws and healing abutments. To prevent the application of excessive or insufficient torque, dentists must know how much torque can be applied by their fingers ${ }^{12,13}$. Many factors are considered to influence the maximum torque (MT) with manually operated implant drivers, including muscle strength and proficiency of the operator. These specifically refer not only to such intrinsic factors as operator's grip strength and finger 
strength but also to such extrinsic factors as the use or nonuse of gloves.

Research into the correlations between these factors and the consistency with which operators can generate the recommended torque when using their fingers is limited. The purpose of this study was to identify the factors that affect the torque value of manual drivers, which is applied prior to fastening an implant with a torque wrench.

\section{Materials and methods}

\section{Participants and study design}

Implant treatment has become increasingly common in recent years and is now included in the National Examination for Dentists, highlighting the importance of teaching young dentists how to operate using manual implant drivers. Nine male and 7 female dentists with an average age of 30 years and clinical experience of less than 10 years participated in this study. They joined the study voluntarily after being provided with the details of its procedures and goals. Evaluations were based on the MT and a target torque (TT) of $15 \mathrm{~N}$, and the participants' consistency in achieving these was assessed. The grip and finger strength of the participants were measured to investigate differences in physical ability among them. The research design was approved by the Medical Ethics Committee of the Showa University School of Dentistry (Approval No. 2014-032).

\section{Measurement of torque and equipment}

Feedback on the manual manipulation of an implant driver is influenced by the diameter and shape of the hand grip. We selected two manual implant drivers for this study: the UniGrip Driver (length, $20 \mathrm{~mm}$; Brånemark System; Nobel Biocare, Göteborg, Sweden) and the Carrier Hex Driver (length, 0.050 in; Zimmer Biomet, Tokyo, Japan); these were labeled and hereafter referred to as "Nobel" and "Zimmer," respectively. Both are used in the Showa University Dental Hospital and have a large share of the worldwide market. The driver hand grip diameter and screw pitch of Nobel were 9 and $1.2 \mathrm{~mm}$, respectively, whereas those of Zimmer were 11 and $1.7 \mathrm{~mm}$, respectively (Figure $1 \mathrm{~A}-\mathrm{D}$ ). These were set to a torque gauge (BTG $60 \mathrm{CN}-$ $\mathrm{S}$; Tohnichi, Tokyo, Japan), as shown in Figure 2A and $\mathrm{B}$. The participants were asked to hold and tighten the hand grip set to the torque gauge with their thumb and index finger. The torque gauge used in this study is commonly applied for measuring small torques, inspection, and tightening. The size and weight of the torque gauge make it portable, and the gauge can record torque in both clockwise (CW) and counterclockwise (CCW) directions. The participants were able to see the torque they had applied. The torque gauge's range of measurement was $6-60 \mathrm{~N}$, and its accuracy was high at $\pm 2 \%$. In dental operations, tightening of the implant screw is conducted in two environments: chairside and laboratory treatments. In general, gloves are worn in chairside treatment but not in laboratory treatment. Measurements were therefore taken both when the participants were wearing gloves and when they were not. CLINGLOVES eco gloves (K.O. Dental, Tokyo, Japan) were used throughout the study.

\section{Maximum attainable torque}

Each participant was asked to apply their maximum force using Nobel and Zimmer. Both manual implant drivers were used to tighten the screw in the $\mathrm{CW}$ and $\mathrm{CCW}$ directions with or without the use of gloves. Measurements were conducted every $3 \mathrm{~min}$ under these eight conditions (Type of Driver [Nobel or Zimmer] $\times$ Rotational Direction $[\mathrm{CW}$ or $\mathrm{CCW}]$ With and Without Gloves), giving 32 measurements in

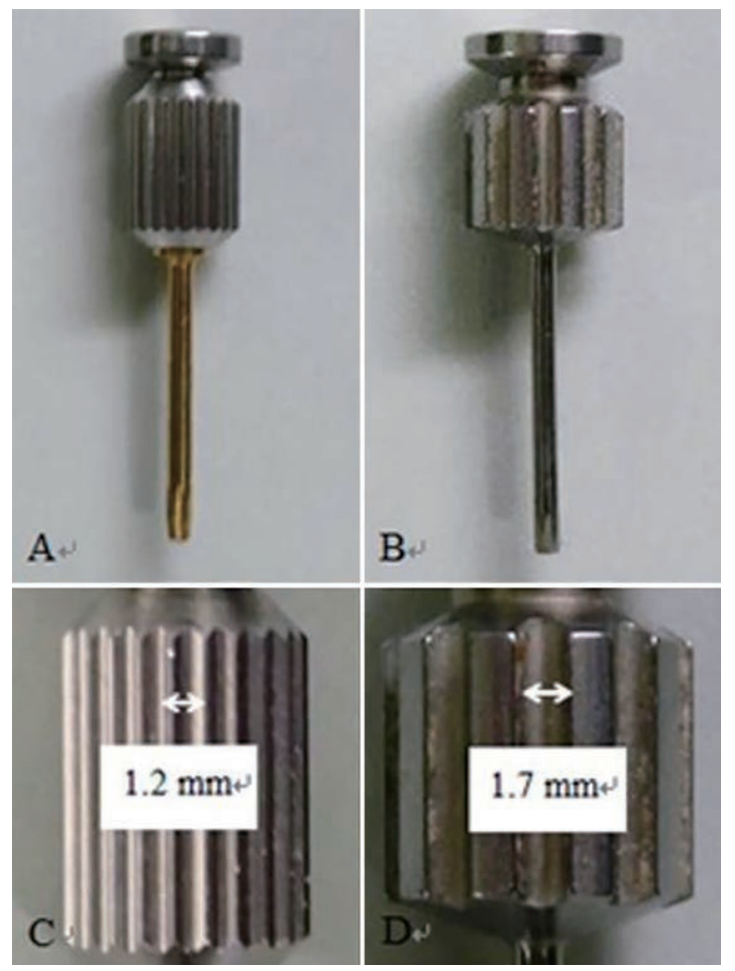

Fig. 1. General view and thread pitch of the manual implant drivers used in this study

A : General view of Nobel Biocare's UniGrip.

B : General view of Zimmer's Carrier Hex.

C : Thread pitch of Nobel Biocare's UniGrip.

D : Thread pitch of Zimmer's Carrier Hex. 


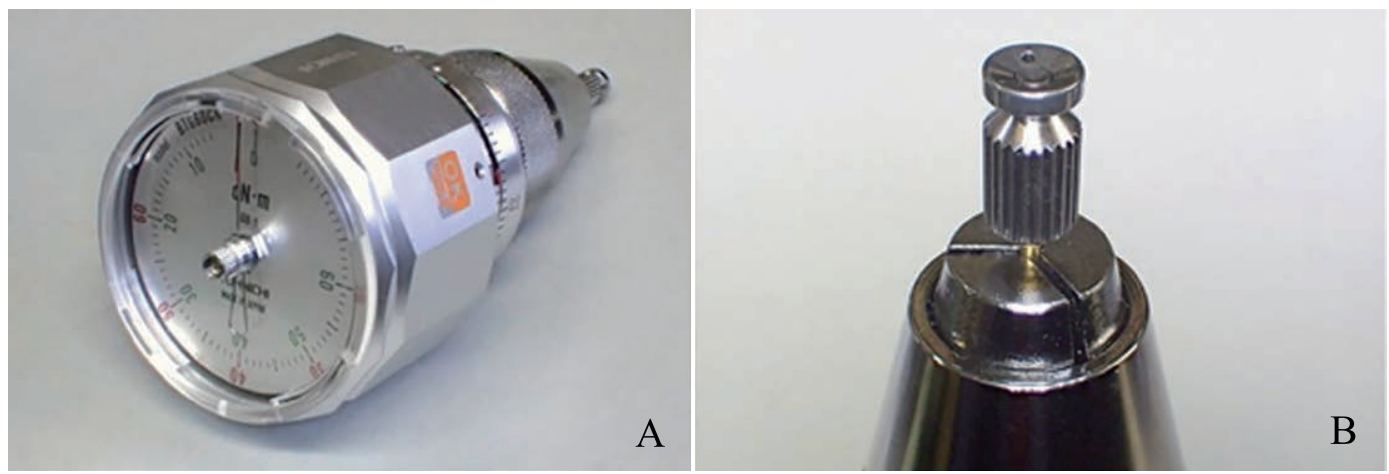

Fig. 2. General view of the torque gauge and manual implant driver attached
A : General view of the torque gauge. The bottom of the torque gauge has a gauge to measure the torque.
B : Manual implant driver set to the torque gauge.

total. A Latin square design was adopted to remove the influence of participant fatigue.

\section{TT of $15 \mathrm{~N}$}

The participants were asked to manually tighten the implant using Nobel and to stop when they believed they had achieved a torque of $15 \mathrm{~N}$. Measurements were made four times at intervals of 3 min under two conditions (CW and $\mathrm{CCW}$ directions), resulting in a total of eight measurements.

\section{Measurement of grip and finger strength}

A digital hand grip meter (MCZ-5041; Macros, Tokyo, Japan) was used to determine the participants' grip and finger strength. Grip strength was measured with the meter gripped by the hand, whereas finger strength was measured with the meter gripped by the thumb and index finger. Measurements were repeated four times at intervals of $3 \mathrm{~min}$.

\section{Statistical analysis}

The Shapiro-Wilk test was used to determine the normality of MT, after which, a three-way analysis of variance was run using the following factors: the type of manual implant driver (Nobel or Zimmer), rotational direction ( $\mathrm{CW}$ or $\mathrm{CCW}$ ), and gloves (with or without). Two-way analysis of variance was conducted using the baseline data and data at one day, week, and month after training to confirm the effectiveness of the training. Pearson's analysis was used to investigate the correlation between MT and grip strength and that between MT and finger strength. All analyses used a significance level of 0.05 and were performed using Statistical analysis software (PASW Statistics 23; IBM, Tokyo, Japan).

\section{Results}

\section{Maximum attainable torque}

A graphical representation of the maximum attainable torque under the eight conditions described above is given in Figure 3. The MT observed with the use of Zimmer, rotation in the CW direction, and use of gloves was significantly higher $(p<0.05)$ than that detected with the use of Nobel, rotation in the CCW direction, and nonuse of gloves. The highest MT was $31.5 \mathrm{~N}$, which was recorded for a male participant performing a $\mathrm{CW}$ rotation using Zimmer with gloves; the lowest MT was $7.0 \mathrm{~N}$, which was recorded for a female participant performing a $\mathrm{CCW}$ rotation using Nobel without gloves.

\section{TT of $15 \mathrm{~N}$}

Measurements of the $15 \mathrm{~N}$ TT are shown in Figure 4. The average torque of the male participants was greater than $15 \mathrm{~N}$ in both rotational directions, whereas that of the female participants was less than $15 \mathrm{~N}$.

\section{Grip strength and finger strength}

The average grip and finger strength of the male participants were 42.4 and $10.3 \mathrm{~kg}$, respectively, whereas those of the female participants were 22.4 and $6.7 \mathrm{~kg}$, respectively. All measured grip and finger strength data, as well as the correlations between them, are shown in Figure 5. Significant positive correlations were found between grip strength and MT $(r=0.79, p<0.01)$ and between finger strength and MT $(r=0.64, p<0.01)$. 

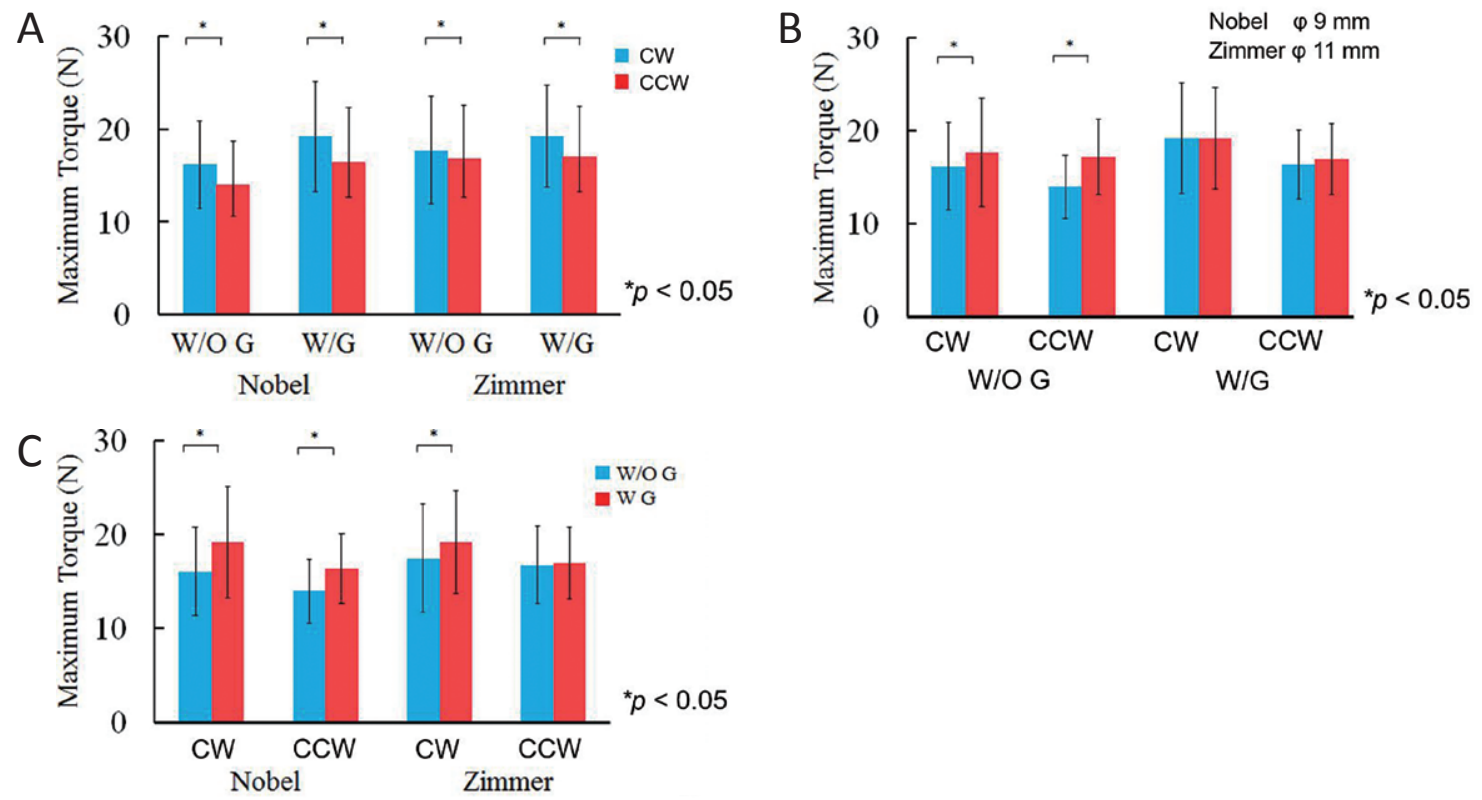

Fig. 3. Maximum torque values under different conditions

A : Comparison of torque values obtained in the clockwise (CW) direction and those obtained in the counterclockwise (CCW) direction.

B : Comparison of torque values obtained using Nobel Biocare's UniGrip with a diameter of 9 $\mathrm{mm}$ and those obtained using Zimmer's Carrier Hex with a diameter of $11 \mathrm{~mm}$.

C : Comparison of torque values obtained with gloves (W/G) and those obtained without gloves (W/O G).

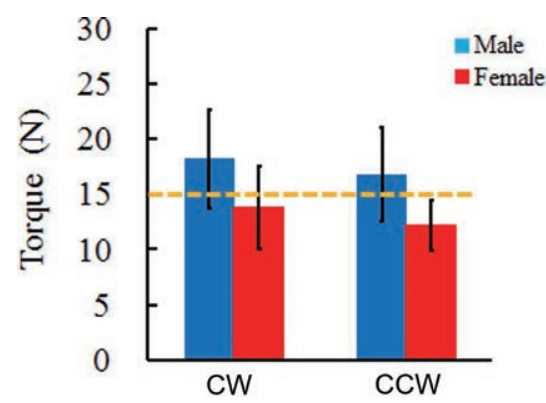

Fig. 4. Comparison of torque values obtained relative to the target torque of $15 \mathrm{~N}$ between male and female participants

\section{Discussion}

\section{Factors related to $M T$}

The MT was measured taking the following factors into account: type of the manual implant driver (Nobel or Zimmer), use or nonuse of gloves, rotational direction $(\mathrm{CW}$ or $\mathrm{CCW})$, as well as each participant's sex (male or female), grip strength, and finger strength. When gloves were not worn by the participants, the MT applied with Zimmer was significantly higher $(p<0.05)$ than that applied with Nobel. On the other hand, when gloves were worn by the participants, the MT applied with Nobel was significantly higher $(p<0.05)$ than that applied with
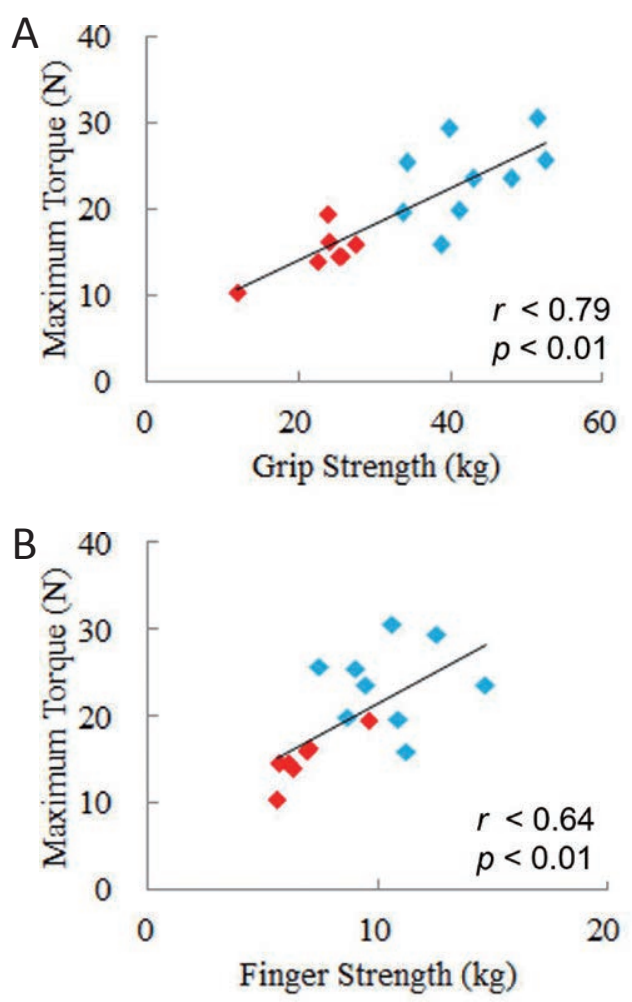

Fig. 5. Correlations between maximum attainable torque, grip strength, and finger strength

A : Relationship between the maximum attainable torque and grip strength.

B : Relationship between the maximum attainable torque and finger strength. 
Zimmer. For measurements conducted with the use of gloves, the thickness of the glove material was added to that of the fingers and grip. The effect of wearing gloves was greater with Nobel because the diameter of its grip is smaller than that of the Zimmer grip. Except for when Zimmer was used without gloves, the MT was higher in all rotational directions. Furthermore, the MT applied by the male participants, who had greater finger and grip strength, was significantly higher than that applied by the female participants. The positive correlations found between MT and finger strength and between grip strength and MT suggest that MT is strongly influenced by the operator's muscles.

\section{Measurement of the TT}

In both rotational directions, the average torque of the male participants greatly exceeded $15 \mathrm{~N}$, whereas that of the female participants was below the TT of $15 \mathrm{~N}$. Some reports have indicated that manual drivers produce inconsistent closing torque values ${ }^{10,13-15}$. In this study, the comparison between the male and female participants revealed a substantial standard deviation, which suggested that the female participants found it difficult to gauge their own torque.

In clinical situations, the prosthetic screw is fastened first with a manual driver and then with a torque wrench at the specified torque value. Therefore, this study focused on the torque value obtained by manual fastening, which is performed first. Future studies should examine the factors that affect the torque value when a torque wrench is used.

\section{Conclusions}

In this study, we investigated the MT applied with manual operation of an implant driver and the consistency with which the recommended torque could be achieved. Our findings indicate that (1) the MT is influenced by the diameter of the manual implant driver, use of gloves, rotation direction, finger strength, and grip strength and (2) dentists should monitor their ability to consistently achieve the recommended torque for implant drivers.

\section{Declaration of competing interests}

The authors have no direct or indirect financial interest in the products or information described in the article.

\section{Funding}

This study was supported by the Grant-in-Aid for Scientific Research (C) from the Ministry of Education, Culture, Sports, Science, and Technology of Japan (Grant No. 25463016).

\section{Acknowledgments}

The authors would like to express their deep appreciation to the teaching staff of the Geriatric Dentistry program at Showa University Dental Hospital for their help and cooperation. A partial summary of this thesis was presented at the 46th Annual Meeting of the Japanese Society of Oral Implantology in Nagoya, Japan, in September 2016.

\section{References}

1. Zarb GA, Schmitt A. The longitudinal clinical effectiveness of osseointegrated dental implants: the Toronto study. Part I: surgical results. J Prosthet Dent. 1990;63:451-457.

2. Zarb GA, Schmitt A. The longitudinal clinical effectiveness of osseointegrated dental implants: the Toronto study. Part II: the prosthetic results. J Prosthet Dent. 1990;64:53-61.

3. Zarb GA, Schmitt A. The longitudinal clinical effectiveness of osseointegrated dental implants: the Toronto study. Part III: problems and complications encountered. J Prosthet Dent. 1990;64:185-194.

4. Wicks RA, deRijk WG, Windeler AS. An evaluation of fit in osseointegrated implant components using torque /turn analysis. J Prosthodont. 1994;3:206-212.

5. Jemt T, Book K. Prosthesis misfit and marginal bone loss in edentulous implant patients. Int $\mathrm{J}$ Oral Maxillofac Implants. 1996;11:620-625.

6. Kan JY, Rungcharassaeng K, Bohsali K, et al. Clinical methods for evaluating implant framework fit. $J$ Prosthet Dent. 1999;81:7-13.

7. Wee AG, Aquilino SA, Schneider RL. Strategies to achieve fit in implant prosthodontics: a review of the literature. Int J Prosthodont. 1999;12:167-178.

8. Ekfeldt A, Carlsson GE, Borjesson G. Clinical evaluation of single-tooth restorations supported by osseointegrated implants: a retrospective study. Int $J$ Oral Maxillofac Implants. 1994;9:179-183.

9. Kallus T, Bessing C. Loose gold screws frequently occur in full-arch fixed prostheses supported by osseointegrated implants after 5 years. Int $J$ Oral Maxillofac Implants. 1994:9:169-178.

10. Goheen KL, Vermilyea SG, Vossoughi J, et al. Torque generated by handheld screwdrivers and mechanical torquing devices for osseointegrated implants. Int $\mathrm{J}$ Oral Maxillofac Implants. 1994;9:149-155.

11. Stroosnijder E, Gresnigt MM, Meisberger EW, et al. Loss of accuracy of torque wrenches due to clinical use and cleaning procedure: short communication. Int 
J Prosthodont. 2016;29:253-255.

12. Sato $Y$, Osawa T, Kitagawa N. Device for easily measuring of the torque of an implant hand driver. Springerplus. 2016;5:1374. (accessed 2021 May 31) Available from: https://www.ncbi.nlm.nih.gov/pmc/articles/ PMC4993727/pdf/40064_2016_Article_3071.pdf

13. Kanawati A, Richards MW, Becker JJ, et al. Measurement of clinicians' ability to hand torque dental implant components. J Oral Implantol. 2009;35:185-188.

14. Gross M, Kozak D, Laufer BZ, et al. Manual closing torque in five implant abutment systems: an in vitro comparative study. J Prosthet Dent. 1999;81:574-578.

15. Sameera $Y$, Rai R. Tightening torque of implant abutment using hand drivers against torque wrench and its effect on the internal surface of implant. $J$ Indian Prosthodont Soc. 2020;20:180-185. 